\title{
MODAL CHARACTERIZATION OF SANDWICH SKEW PLATES
}

\author{
Dhotre PAVAN KUMAR ${ }^{*}$, Chikkol V. SRINIVASA $^{* *}$ \\ *Research Scholar, Department of Mechanical Engineering, GM Institute of Technology,Davangere, Visvesvaraya Technological University, \\ Jnana Sangama, VTU Main Rd, Machhe, Belagavi, Karnataka 590018, India \\ **Head, Department of Mechanical Engineering, GM Institute of Technology, Davangere, Visvesvaraya Technological University, \\ Jnana Sangama, VTU Main Rd, Machhe, Belagavi, Karnataka 590018, India \\ pavankumar-gmitrs@gmit.ac.in, srinivasacv@gmit.ac.in
}

received 14 March 2021, revised 26 June 2021, accepted 1 July 2021

\begin{abstract}
The current work focuses on the experimental and finite element free vibration studies of laminated composite sandwich skew plates. The comparison was made between the experimental values obtained by the Fast Fourier transform (FFT) analyzer and a finite element solution obtained from CQUAD8 finite element of The MacNeal-Schwendler Corporation (MSC) / NASA STRucture Analysis (NASTRAN) software. The influence of parameters such as aspect ratio (AR) (a/b), skew angle (a), edge condition, laminate stacking sequence, and fiber orientation angle $\left(\theta^{\circ}\right)$ on the natural frequencies of sandwich skew plates was studied. The values obtained by both the finite element and experiment approaches are in good agreement. The natural frequencies increase with an increase in the skew angle for all given ARs.
\end{abstract}

Keywords: natural frequency, non-dimensional frequency parameter $\left(\mathrm{K}_{\mathrm{f}}\right)$, antisymmetric laminate, fiber orientation angle, skew angle

\section{INTRODUCTION}

Due to the reduced weight and high stiffness, sandwich structures have a wide area of applications in engineering science. With anisotropy and considering all parameters involved in the sandwich structure, it is difficult to evaluate the dynamic response analytically. In the literature review, various theories, methods, and techniques were cited (Pavan et al., 2021). However, with the evolution of technology in real engineering applications, it is possible to predict the dynamic response of any structure accurately.

First,an experimental approach to the determination of natural frequencies of sandwich plates was made (Raville et al., 1967). Verification was made (Barkanov et al., 2005) of the numerical results of laminated composite and different sandwich panels with pulse and noncontact laser techniques. Static deformation and free vibration study was conducted on sandwich plates with variable thickness using both numerical and experimental holographic interferometry techniques (Chang et al., 2006). A theoretical and experimental study was conducted on the vibration and acoustical properties of sandwich composite materials (Zhuang, 2006). The vibration testing approach was used to identify the material constants (Lee et al., 2007) and damping characteristics (Berthelot et al., 2008; Maheri et al., 2008; Andena et al., 2012; Petrone et al., 2014; Yang et al., 2014; Abdi et al., 2014) with the help of experimentally obtained natural frequency values of laminated composite sandwich plates. Dynamic behavior of honeycomb sandwich plates was investigated considering the effect of cell size (Adarsh Kumar et al., 2015) and other parameters (Mondal et al., 2015; Rezvani et al., 2018; Prasad et al., 2018; Benjeddou et al., 2019; Arunkumar et al., 2020; Zhicheng et al., 2020) of the honeycomb core. Free vibration and transient dynamic analyses of functionally graded sandwich plates are investigated (Jun Liu et al., 2021) using the scaled boundary finite element method (SBFEM). Nu- merical studies were presented (Vinayak et al., 2020) on skew composite laminated and sandwich plates under temperature and moisture concentration effects; the laminated sandwich plate is subjected to hygrothermal conditions (Aman et al., 2020) and composite sandwich plates with three-dimensional stress recovery (Su Bin Lee et al., 2020). A review on the analysis of laminated composite and sandwich structures under hygrothermal conditions addressing bending, vibration, buckling, post-buckling, transient, dynamic, and impact studies were made (Aman et al., 2019). An extensive survey on the analysis of sandwich FGM structures under different loading conditions, effects of porosities, hygrothermal loadings, and structures resting on elastic foundations was made (Aman et al., 2020).

A vast amount of literature was reported in detail on free vibration studies for laminated composite skew sandwich plates using analytical and numerical approaches. Limited literature was found related to detailed experimental work on the dynamic response of laminated composite sandwich skew plates. Literature on finite element solutions validated by the experimental method is very scarce. The present paper is an attempt to address this issue in some detail. Fast Fourier transform (FFT) analyzer is an instrument best suited for dynamic applications. In the current work, an FFT analyzer is used for the prediction of natural frequencies of sandwich skew plates.

\section{TEST SPECIMEN PREPARATION}

The sandwich skew panels were prepared using glass/epoxy laminated composites as face sheets and aluminum honeycomb as core materials. Aluminum honeycomb (Al-3003) panels are 
used as the core with a cell size of $6.35 \mathrm{~mm}$, foil thickness of 50 microns, and height of $6 \mathrm{~mm}$. Laminated glass/epoxy reinforced polymer composites are used as face sheets. Unidirectional glass fibers for $\left[ \pm 0^{\circ} / \mathrm{Core} / \pm 0^{\circ}\right],\left[ \pm 90^{\circ} / \mathrm{Core} / \pm 90^{\circ}\right]$ and bidirectional glass fibers for $\left[ \pm 45^{\circ} / \mathrm{Core} / \pm 45^{\circ}\right]$, $\left[\left(0^{\circ} / 90^{\circ}\right)_{5} / \mathrm{Core} /\left(0^{\circ} / 90^{\circ}\right)_{5}\right]$ of $220 \mathrm{gsm}$ and Lapox L-12 (Epoxy) along with Lapox K-6 hardener were used in fabricating the laminates. Continuous hand lay-up technique was employed for fabricating the sandwich plates, during which the excess resin was removed from the laminate by steel roller. The sandwich laminates are cured at room temperature for a period of $48 \mathrm{~h}$ placing weights over them. The test specimens were prepared with fiber weight percentage 50:50 according to relevant American Society for Testing and Materials ASTM standards.

The material constants for aluminum honeycomb core are $E_{x}=E_{y}=0.4043 \mathrm{MPa}, \quad E_{z}=941.0802 \mathrm{MPa}, \quad G_{x y}=0.0546 \mathrm{MPa}$, $\mathrm{G}_{\mathrm{yz}}=110.3007 \mathrm{MPa}, \quad \mathrm{G}_{\mathrm{xz}}=73.5338 \mathrm{MPa}, \quad \rho=48.37 \mathrm{~kg} / \mathrm{m}^{3}$, $v_{x}=0.994 \approx 1$, and $v_{y}=v_{z}=0.001$ and evaluated using the formulae presented (Rajkumar S., etal., 2014). ASTM Standard D3039/D3039M (2006) are imposed while evaluating the material properties of laminated glass/epoxy composite panels. The material constants $E_{1}$ and $E_{2}$ were evaluated experimentally on an average of three trials using Instron Universal Testing Machine at the Central Institute of Plastics Engineering and Technology (CIPET), Mysore, Karnataka, India. Strain gauges were used for calculating strains in longitudinal (along the loading) and transverse (right angles to the loading) directions. The ratio of measured strains concludes the value of Poisson's ratio $v_{12}$ within the elastic range. The average of three experimental trials wasmade during the determination of the value of Poisson's ratio $v_{12}$. The standard equation of shear modulus presented in Jones(1999) was implemented in calculations and the material properties imposed are given in Tab. 1. The aspect ratio (AR) of the test specimens varied from 1.0 to 2.5 and the skew angle varied from $0^{\circ}$ to $45^{\circ}$.

Tab. 1. Material constants of E-glass fiber.

\begin{tabular}{|c|c|c|c|}
\hline Parameter & Mean & Standard & \%Error \\
\hline$E_{1}[\mathrm{GPa}]$ & 38.07 & 1.205 & 0.048 \\
\hline$E_{2}[\mathrm{GPa}]$ & 8.1 & 0.271 & 0.033 \\
\hline $\mathbf{G}_{12}[\mathrm{GPa}]$ & 3.05 & 0.095 & 0.039 \\
\hline $\mathbf{V}_{12}$ & 0.22 & 0.036 & 0.015 \\
\hline$\rho_{12}\left[\mathrm{~kg} / \mathrm{m}^{3}\right]$ & \multicolumn{3}{|c}{2,200} \\
\hline
\end{tabular}

\section{EXPERIMENTAL SETUP}

The arrangement for conducting the experimentation is shown in Fig. 1. The test specimen was held in the fixtures, imposing Clamped-Free-Clamped-Free and Clamped-Free-Free-Free edge conditions. A piezoelectric accelerometer sensor was placed at the center of the test specimen using glue and was connected to the FFT analyzer (signal conditioning and amplifying unit). An impact hammer was also connected to the FFT analyzer, dedicated to exciting the test specimen on selected points five times. For each test specimen, five trials were made and an average value was adopted.

Soon, the impact hammer excites the test specimen with a strike (impact); the accelerometer sensor captures the vibration signals and exports them to the FFT analyzer for further pro- cessing. The FFT analyzer gives the output in terms of Frequency Response Function (FRF) using the pulse lab software. It was ensured that the strike of the impact hammer was normal to the test specimen's surface and no other sources of excitations (surrounding and floor vibrations nearby) were present other than the impact hammer.

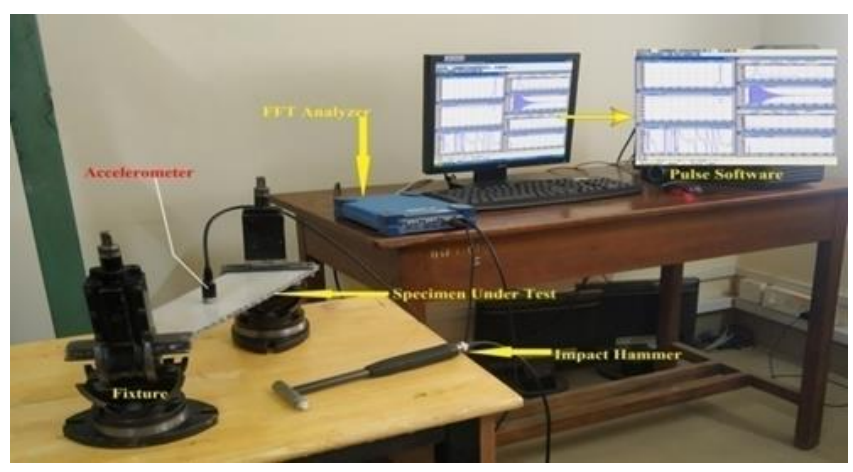

Fig. 1. Experimental Setup.

\section{FINITE ELEMENT ANALYSIS}

MSC/NASTRAN software package was employed for finite element analysis in obtaining the first three fundamental frequencies of skew sandwich plates. Eight-noded isoparametric curved shell elements, i.e., CQUAD8 and CQUAD4, are used in the analysis. A study (Pavan et al., 2020) disclosed that the CQUAD8 element produces more converging and accurate results than the CQUAD4 element. Accordingly, the CQUAD8 element was employed in the present study. To evaluate the real eigenvalues and eigenvectors, the Lanczos method of extraction was imposed for accurate results.

\section{RESULTS AND DISCUSSION}

The current work focuses on the influence of AR, skew angle, laminate stacking sequence, and edge conditions on the sandwich skew plates. To obtain higher natural frequencies of sandwich skew plates, considering the influence of tc/tf and $a / h$ as discussed (Pavan et al., 2021) and minimizing the production cost, twenty-one layer, antisymmetric, angle-ply, and cross-ply laminated sandwich plates are designed and prepared. The thickness ratio of core to face sheet is kept constant in the whole study as $\mathrm{tc} / \mathrm{tf}=6$ and the ratio of length overall thickness $\mathrm{a} / \mathrm{h}$ is varied to $12.5(\mathrm{a} / \mathrm{b}=1.0), 18.75(\mathrm{a} / \mathrm{b}=1.5), 25(\mathrm{a} / \mathrm{b}=2.0)$, and 31.25 $(a / b=2.5)$. Two types of edge conditions are implemented in the study i.e., Clamped-Free-Clamped-Free Edge Condition [C-F-C-F] and Clamped-Free-Free-Free Edge Condition [C-F-F-F]. The results from the current study are presented in non-dimensional form using Eq. (1).

$K_{f}=100 \omega a \sqrt{\left(\frac{\rho}{E_{1}}\right)_{f}}$

First, three natural frequencies are extracted from experiments in non-dimensional form. The experimental values are compared with finite element values. The results are tabulated for the C-F-C-F edge condition in Tab. 2 and also graphically presented in Figs. 2-5. The mode shapes are presented in Tab. 3. 
The results are tabulated for the C-F-F-F edge condition in Tab. 4 and also graphically presented in Figs. 6-9. The mode shapes are presented in Tab. 5.

Tab. 2. Natural frequencies of Clamped-Free-Clamped-Free laminated composite sandwich skew plates

\begin{tabular}{|c|c|c|c|c|c|c|c|c|}
\hline \multicolumn{9}{|c|}{ Skew Angle (a) $0^{0}$} \\
\hline \multirow{3}{*}{$\begin{array}{l}\text { Mo. } \\
\text { No. }\end{array}$} & \multicolumn{8}{|c|}{ Aspect Ratio (alb) } \\
\hline & \multicolumn{2}{|c|}{1.0} & \multicolumn{2}{|c|}{1.5} & \multicolumn{2}{|c|}{2.0} & \multicolumn{2}{|c|}{2.5} \\
\hline & Exp & Num & Exp & Num & Exp & Num & Exp & Num \\
\hline \multicolumn{9}{|c|}{ Angle ply $\left[\left(+0^{0} /-0^{0}\right)_{5} /\right.$ Core $\left./\left(+0^{\circ} /-0^{0}\right)_{5}\right]$} \\
\hline 1 & 7.334 & 7.408 & 5.539 & 5.594 & 4.372 & 4.415 & 3.579 & 3.623 \\
\hline 2 & 9.783 & 9.892 & 9.156 & 9.262 & 8.759 & 8.886 & 8.482 & 8.628 \\
\hline 3 & 26.069 & 26.439 & 22.973 & 23.283 & 20.185 & 20.572 & 17.862 & 18.239 \\
\hline \multicolumn{9}{|c|}{ Angle ply $\left[\left(+45^{\circ} /-45^{0}\right)_{5} /\right.$ Core $\left./\left(+45^{\circ} /-45^{\circ}\right)_{5}\right]$} \\
\hline 1 & 4.945 & 4.995 & 3.392 & 3.425 & 2.546 & 2.571 & 2.026 & 2.047 \\
\hline 2 & 12.260 & 12.442 & 12.273 & 12.433 & 12.223 & 12.403 & 11.545 & 11.765 \\
\hline 3 & 20.919 & 21.301 & 16.969 & 17.254 & 13.826 & 14.107 & 12.125 & 12.377 \\
\hline & & & le ply [(+ & $0 \%-90 \%)_{5}$ & ore/ $(+90$ & $\left.\left.90^{\circ}\right)_{5}\right]$ & & \\
\hline 1 & 4.082 & 4.123 & 3.183 & 3.214 & 2.139 & 2.160 & 1.721 & 1.738 \\
\hline 2 & 8.569 & 8.678 & 8.288 & 8.390 & 7.989 & 8.086 & 7.837 & 7.945 \\
\hline 3 & 19.468 & 19.764 & 16.398 & 16.699 & 12.144 & 12.376 & 10.077 & 10.259 \\
\hline & & & ngle ply & $(0 \% / 900)_{5} /$ & ore/ $(0 \% / 9$ & & & \\
\hline 1 & 6.278 & 6.340 & 4.559 & 4.604 & 3.535 & 3.570 & 2.874 & 2.902 \\
\hline 2 & 9.379 & 9.488 & 8.817 & 8.939 & 8.509 & 8.611 & 8.279 & 8.387 \\
\hline 3 & 24.409 & 24.813 & 20.770 & 21.142 & 17.800 & 18.119 & 15.406 & 15.667 \\
\hline & & & & w Angle & 1) $15^{\circ}$ & & & \\
\hline & & & ngle ply & $+0 \%-00)_{5}$ & ore/ $\left(+0^{0}\right)$ & & & \\
\hline 1 & 6.848 & 6.917 & 4.736 & 4.783 & 3.917 & 3.955 & 3.159 & 3.190 \\
\hline 2 & 10.530 & 10.649 & 10.510 & 10.637 & 9.652 & 9.770 & 9.429 & 9.543 \\
\hline 3 & 25.851 & 26.269 & 21.918 & 22.235 & 19.428 & 19.752 & 17.005 & 17.307 \\
\hline & & & le ply [(+ & $5 \%-45)_{5}$ & ore/ (+45 & $\left.\left.45^{0}\right)_{5}\right]$ & & \\
\hline 1 & 5.208 & 5.259 & 3.847 & 3.883 & 2.716 & 2.743 & 2.169 & 2.189 \\
\hline 2 & 12.135 & 12.274 & 11.419 & 11.555 & 11.855 & 11.987 & 11.691 & 11.833 \\
\hline 3 & 21.879 & 22.206 & 18.814 & 19.088 & 14.700 & 14.933 & 12.428 & 12.567 \\
\hline & & & le ply [( & $0 \%-900)_{5}$ & ore/ $(+90$ & $\left.\left(90^{\circ}\right)_{5}\right]$ & & \\
\hline 1 & 4.163 & 4.204 & 2.916 & 2.945 & 2.158 & 2.179 & 1.730 & 1.747 \\
\hline 2 & 9.026 & 9.138 & 8.968 & 9.076 & 8.437 & 8.542 & 8.250 & 8.334 \\
\hline 3 & 19.968 & 20.289 & 16.065 & 16.334 & 12.554 & 12.730 & 10.480 & 10.630 \\
\hline & & & ngle ply & $\left.\left(0^{0} / 90^{\circ}\right)_{5}\right)$ & ore/ $(0 \% 9$ & & & \\
\hline 1 & 6.021 & 6.080 & 4.177 & 4.219 & 3.321 & 3.354 & 2.669 & 2.695 \\
\hline 2 & 10.075 & 10.195 & 10.183 & 10.302 & 9.342 & 9.455 & 9.129 & 9.239 \\
\hline 3 & 24.385 & 24.722 & 20.478 & 20.824 & 17.560 & 17.833 & 15.118 & 15.344 \\
\hline & & & & w Angle & 1) $30^{\circ}$ & & & \\
\hline & & & ngle ply & $+0 \%-00)_{5}$ & ore $/(+0 \%$ & & & \\
\hline 1 & 6.177 & 6.239 & 4.354 & 4.397 & 3.250 & 3.282 & 2.544 & 2.569 \\
\hline 2 & 12.335 & 12.483 & 11.810 & 11.952 & 11.362 & 11.495 & 10.825 & 10.948 \\
\hline 3 & 24.987 & 25.356 & 21.502 & 21.824 & 18.421 & 18.678 & 16.105 & 16.346 \\
\hline & & & le ply [( & $5 \%-45)_{5}$ & ore/ $(+45$ & $\left.\left.45^{0}\right)_{5}\right]$ & & \\
\hline 1 & 6.035 & 6.094 & 4.227 & 4.268 & 3.211 & 3.243 & 2.576 & 2.601 \\
\hline 2 & 11.965 & 12.118 & 11.418 & 11.549 & 11.048 & 11.178 & 10.785 & 10.919 \\
\hline 3 & 24.653 & 25.025 & 20.351 & 20.655 & 16.954 & 17.206 & 14.350 & 14.556 \\
\hline & & & le ply [( & $0 \%\left(-90^{\circ}\right)_{5}$ & ore $/(+90$ & $\left.\left(90^{\circ}\right)_{5}\right]$ & & \\
\hline 1 & 4.472 & 4.516 & 3.021 & 3.051 & 2.254 & 2.277 & 1.789 & 1.807 \\
\hline 2 & 10.515 & 10.632 & 10.139 & 10.252 & 9.778 & 9.889 & 9.200 & 9.312 \\
\hline 3 & 21.675 & 21.993 & 16.981 & 17.227 & 13.815 & 14.032 & 11.952 & 12.128 \\
\hline & & & ngle ply & $\left.\left(0^{0} / 90^{\circ}\right)_{5}\right)$ & ore/ $(0 \%$ & & & \\
\hline 1 & 5.820 & 5.877 & 4.031 & 4.070 & 2.999 & 3.029 & 2.367 & 2.390 \\
\hline 2 & 12.115 & 12.260 & 11.711 & 11.834 & 11.289 & 11.425 & 10.770 & 10.897 \\
\hline 3 & 24.875 & 25.277 & 20.734 & 21.030 & 17.529 & 17.786 & 15.388 & 15.619 \\
\hline & & & & w Angle & 1) $45^{\circ}$ & & & \\
\hline & & & ngle ply & $+0 \%-00)_{5}$ & ore $/\left(+0^{0}\right)$ & & & \\
\hline 1 & 5.760 & 5.818 & 3.912 & 3.951 & 2.843 & 2.871 & 1.989 & 2.009 \\
\hline 2 & 15.066 & 15.237 & 13.846 & 14.006 & 12.535 & 12.686 & 10.768 & 10.885 \\
\hline 3 & 25.448 & 25.826 & 20.758 & 21.055 & 15.564 & 15.785 & 11.041 & 11.203 \\
\hline & & & le ply [( & $5 \%-45)_{5}$ & ore/ $(+45$ & $\left.\left.45^{0}\right) 5\right]$ & & \\
\hline 1 & 7.104 & 7.173 & 5.058 & 5.108 & 3.853 & 3.889 & 3.101 & 3.130 \\
\hline 2 & 12.971 & 13.122 & 11.833 & 11.978 & 11.223 & 11.357 & 10.901 & 11.023 \\
\hline 3 & 28.723 & 29.136 & 24.077 & 24.434 & 19.814 & 20.114 & 16.475 & 16.717 \\
\hline & & & le ply [( & $0 \%-900)_{5}$ & ore $/(+90$ & $\left.\left.90^{0}\right)_{5}\right]$ & & \\
\hline 1 & 5.151 & 5.201 & 3.426 & 3.460 & 2.522 & 2.546 & 2.219 & 2.240 \\
\hline 2 & 13.455 & 13.600 & 12.834 & 12.989 & 11.989 & 12.125 & 11.278 & 11.413 \\
\hline 3 & 24.448 & 24.843 & 17.870 & 18.130 & 13.715 & 13.922 & 12.261 & 12.437 \\
\hline & & & ngle ply & $\left(00 / 90^{\circ}\right)_{5}$ & ore/ $(0 \% / 9$ & & & \\
\hline 1 & 5.952 & 6.011 & 4.005 & 4.044 & 2.920 & 2.949 & 2.287 & 2.310 \\
\hline 2 & 15.478 & 15.667 & 14.637 & 14.810 & 13.421 & 13.587 & 12.016 & 12.158 \\
\hline 3 & 26.647 & 27.030 & 20.933 & 21.247 & 15.823 & 16.048 & 12.564 & 12.745 \\
\hline
\end{tabular}

Tab. 3. Natural frequencies of Clamped-Free-Free-Free laminated composite sandwich skew plates

\begin{tabular}{|c|c|c|c|c|c|c|c|c|}
\hline \multicolumn{9}{|c|}{ Skew Angle (a) $0^{0}$} \\
\hline \multirow{3}{*}{$\begin{array}{l}\text { Mo. } \\
\text { No. }\end{array}$} & \multicolumn{8}{|c|}{ Aspect Ratio (alb) } \\
\hline & \multicolumn{2}{|c|}{1.0} & \multicolumn{2}{|c|}{1.5} & \multicolumn{2}{|c|}{2.0} & \multicolumn{2}{|c|}{2.5} \\
\hline & Exp & Num & Exp & Num & Exp & Num & Exp & Num \\
\hline \multicolumn{9}{|c|}{ Angle ply $\left[(+0 \% /-0)_{5} /\right.$ Core/ $\left.\left(+0^{0} \%-0\right)_{5}\right]$} \\
\hline 1 & .367 & 21.582 & 19.878 & 20.073 & 18.225 & 18.400 & 16.590 & 16.752 \\
\hline 2 & 2.978 & 23.247 & 22.010 & 22.294 & 20.965 & 21.286 & 19.988 & 20.370 \\
\hline 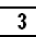 & 35.461 & 36.019 & 40.215 & 40.887 & 38.098 & 38.625 & 35.568 & 36.366 \\
\hline \multicolumn{9}{|c|}{ Angle ply $\left[\left(+450 /-45_{5}\right)_{5} /\right.$ Core $\left./(+45 \%-45)_{5}\right]$} \\
\hline 1 & .336 & 9.526 & 16.445 & 16.609 & 13.870 & 14.010 & 11.789 & 11.905 \\
\hline 2 & 4.832 & 25.164 & 24.810 & 25.126 & 24.729 & 25.042 & 24.681 & 24.969 \\
\hline 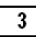 & 39.419 & 39.997 & 35.436 & 36.001 & 31.550 & 32.107 & 28.063 & 28.530 \\
\hline & & & le ply $[(--$ & $200 \%-90)_{5}$ & ore $/\left(+90^{\circ}\right.$ & $\left.900 \%_{5}\right]$ & & \\
\hline 1 & 672 & 848 & 14.399 & 14.542 & 11.868 & 11.984 & 9.981 & 10.081 \\
\hline 2 & .500 & 20.758 & 18.875 & 19.090 & 17.774 & 18.004 & 17.115 & 17.304 \\
\hline 3 & 37.303 & 37.872 & 32.580 & 33.083 & 28.297 & 28.756 & 24.650 & 25.093 \\
\hline & & & Ingle ply & $(00 / 90)_{5} /$ & ree/ $(0 \% / 9$ & (9) & & \\
\hline 1 & 728 & 934 & 18.624 & 18.810 & 16.518 & 16.684 & 4.631 & 14.774 \\
\hline 2 & 22.532 & 22.826 & 21.249 & 21.528 & 20.091 & 20.339 & 19.142 & 19.378 \\
\hline 3 & 41.579 & & 38.541 & 39.293 & 35.829 & & 33.050 & 33.537 \\
\hline & & & & ew Angle & d) $15^{0}$ & & & \\
\hline & & & Ingle ply & $(+00 /-0)_{5}$ & ore $(+0 \%$ & & & \\
\hline 1 & 21.863 & 22.083 & 20.491 & 20.693 & 18.155 & 18.338 & 16.270 & 16.431 \\
\hline 2 & 23.488 & 23.777 & 23.040 & 23.301 & 21.665 & 21.931 & 20.979 & 21.226 \\
\hline 3 & 36.405 & 36.956 & 41.862 & 42.451 & 38.500 & 39.087 & 35.901 & 36.428 \\
\hline & & & gle ply [( & $\frac{450 /-45)_{5}}{4}$ & ore / $\left(+45^{\circ}\right.$ & $\left.\left.-45^{0}\right)_{5}\right]$ & & \\
\hline 1 & 20.018 & 20.218 & 17.927 & \begin{tabular}{|l|l|l|l}
8.105 \\
\end{tabular} & 14.545 & 14.686 & 12.410 & 12.533 \\
\hline 2 & 25.441 & 25.743 & 25.188 & 25.491 & 24.789 & 25.098 & 24.451 & 24.746 \\
\hline 3 & 40.705 & 41.355 & 38.202 & \begin{tabular}{|l|l}
38.790 \\
\end{tabular} & 32.905 & 33.395 & 29.294 & 29.800 \\
\hline & & & gle ply [f & $\frac{100 \%-90)_{5}}{4}$ & ore $/+90^{\circ}$ & $\left.\left.-90^{\circ}\right)_{5}\right]$ & & \\
\hline 1 & 285 & 461 & 15.445 & 15.598 & 12.193 & 12.313 & 10.215 & 10.315 \\
\hline 2 & 21.183 & 21.438 & 20.218 & & 18.650 & 18.868 & 18.013 & 18.221 \\
\hline 3 & 38.597 & 39.159 & \begin{tabular}{|l|l}
34.982 \\
\end{tabular} & \begin{tabular}{|l|l}
35.533 \\
\end{tabular} & 29.253 & 29.663 & 25.479 & 25.859 \\
\hline & & & Angle ply & {$\left[(00 / 90)_{5}\right.$} & orel $(0 \% 9$ & & & \\
\hline 1 & 085 & 94 & 19.233 & 19.423 & 16.600 & 16.760 & 14.528 & 14.666 \\
\hline 2 & 23.045 & & 22.585 & & & & 20.330 & 20.573 \\
\hline 3 & 42.462 & 43.088 & \begin{tabular}{|l|l}
40.493 \\
\end{tabular} & 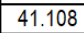 & 36.461 & 36.977 & 33.352 & 33.837 \\
\hline & & & & ew $\mathrm{Ar}$ & $30^{\circ}$ & & & \\
\hline & & & ingle ply & $(+00 \%-00)_{5}$ & ore $(+00)$ & & & \\
\hline 1 & 23.634 & 23.867 & 21.080 & 21.287 & 18.290 & 18.470 & 15.668 & 15.823 \\
\hline 2 & 25.448 & 25.747 & 24.739 & 25.020 & 81 & 24.657 & 24.150 & 24.440 \\
\hline 3 & 39.264 & 39.849 & 43.512 & \begin{tabular}{|l|l|}
44.141 \\
\end{tabular} & 40.348 & 40.924 & 36.453 & 36.981 \\
\hline & & & le ply [6 & $450 /-450_{5}$ & ore $/\left(+45^{\circ}\right.$ & $\left.450_{5}\right]$ & & \\
\hline 1 & 22.285 & 06 & 19.24 & 19.437 & 16.499 & 16.66 & 4.212 & 14.355 \\
\hline 2 & 27.378 & 27.704 & 26.379 & & 25.160 & 25. & 24.125 & 24.412 \\
\hline 3 & 44.825 & 45.273 & 40.682 & 41.280 & 36.629 & 37.166 & 32.971 & 33.445 \\
\hline & & & le ply [- & $600 /-90)_{5}$ & ore $/\left(+90^{\circ}\right.$ & $\left.900{ }^{5}\right]$ & & \\
\hline 1 & & & & & & 13.4 & 12 & 122 \\
\hline 2 & 465 & 23.738 & 22.200 & \begin{tabular}{|l|}
22. \\
\end{tabular} & 21. & 21. & 20.930 & 21.165 \\
\hline 3 & 4 & 4 & 37.288 & & 32 & 32. & 27.998 & 28.403 \\
\hline & & & Angle ply & $\left.\left(0^{0} / 90\right)_{5}\right)$ & ore/ $(0 \% / 9$ & & & \\
\hline & & & 19.977 & & & & & 623 \\
\hline 2 & 25.30 & 25 & 24.673 & 24 & 24.325 & 24 & 4.150 & 24.437 \\
\hline 3 & 43.928 & 44.590 & 42.606 & \begin{tabular}{|l}
43.229 \\
\end{tabular} & 38.342 & 38.912 & 34.588 & 35.121 \\
\hline & & & & ew Ans & $45^{\circ}$ & & & \\
\hline & & & ingle & $(+0 \%$ & ore/ & & & \\
\hline 1 & 555 & 27.829 & 23.433 & 23.670 & 19.112 & 19.300 & 3.043 & 13.172 \\
\hline & 29 & & & & & & 26 & 27.262 \\
\hline 3 & 44.752 & 45.393 & 47.852 & 48.553 & 44.097 & 44.786 & 34.248 & 34.745 \\
\hline & & & , & $450 \%-45^{\circ}$ & ore / $\left(+45^{\circ}\right.$ & $\left.45^{\circ}\right)_{5]}$ & & \\
\hline 1 & 26.785 & 27.054 & 23.186 & 23.419 & 19.934 & 20.133 & 17.786 & 17.960 \\
\hline 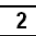 & 31.4 & 31.829 & 29.254 & 29.595 & 27.061 & 27. & 25.944 & 26.240 \\
\hline 3 & 49.350 & 50.058 & 48.321 & 49.005 & 43.598 & 44.227 & 41.424 & 42.022 \\
\hline & & & & & ore/ / + & $\left.(900)_{5}\right]$ & & \\
\hline 1 & 24.688 & 24.930 & 19.634 & 19.829 & 15.678 & 15.833 & 16.158 & 16.319 \\
\hline$\sqrt{n}$ & 28.255 & 28.5 & 27.559 & & 27.185 & 27. & 31.001 & 31.374 \\
\hline 3 & 48.711 & 49.425 & 44.562 & 45.197 & 38.088 & 38.638 & 39.955 & 40.556 \\
\hline & & & Angle ply & & ree/ $(0 \% / 9$ & & & \\
\hline 1 & 27.008 & 27.275 & 22.608 & 22.829 & 18.482 & 18.668 & 15.679 & 15.836 \\
\hline 2 & 30.185 & 30.528 & 30.589 & 30.948 & 30.995 & 31.348 & 31.652 & 32.027 \\
\hline 3 & 48.550 & 49.241 & 49.410 & 50.139 & 42.975 & 43.610 & 39.403 & 39.977 \\
\hline
\end{tabular}




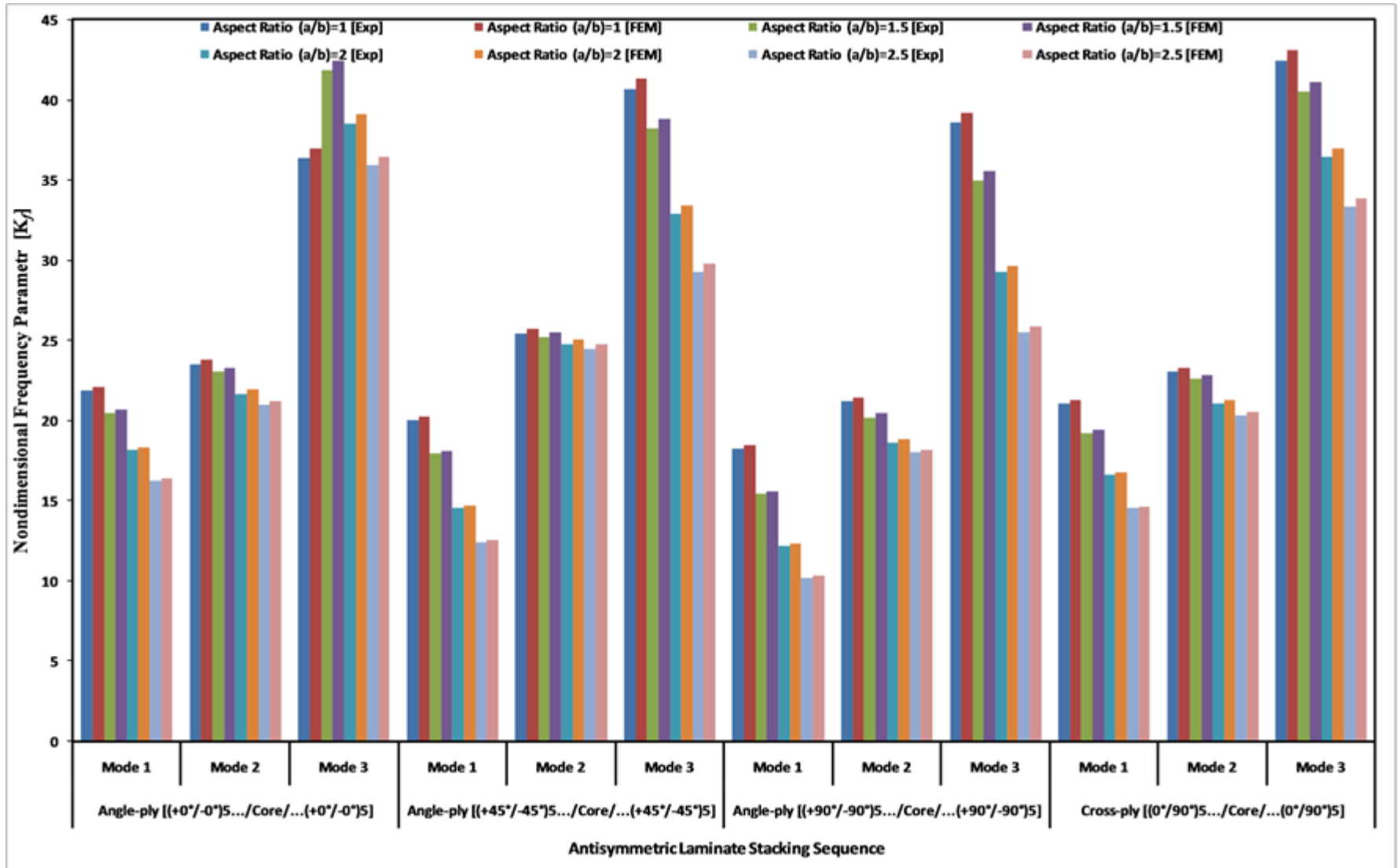

Fig. 2. Variation of $\mathrm{K}_{\mathrm{f}}$ with $\mathrm{AR}(\mathrm{a} / \mathrm{b})$ for $\mathrm{C}-\mathrm{F}-\mathrm{C}-\mathrm{F}$ laminated composite sandwich skew plates with skew angle $\mathrm{a}=0^{\circ}$.

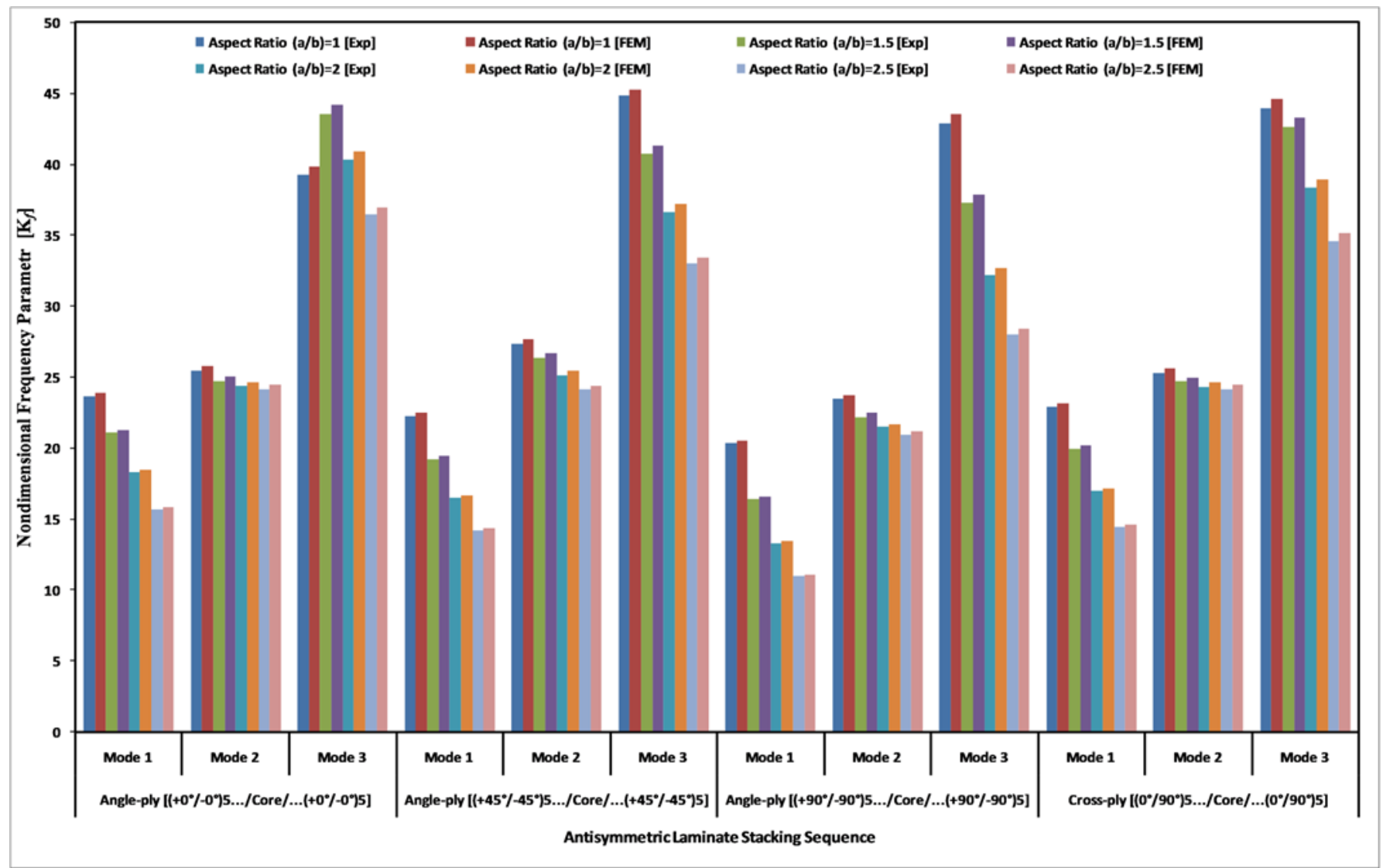

Fig. 3. Variation of $K_{f}$ with $A R(a / b)$ for C-F-C-F laminated composite sandwich skew plates with skew angle $a=15^{\circ}$. 


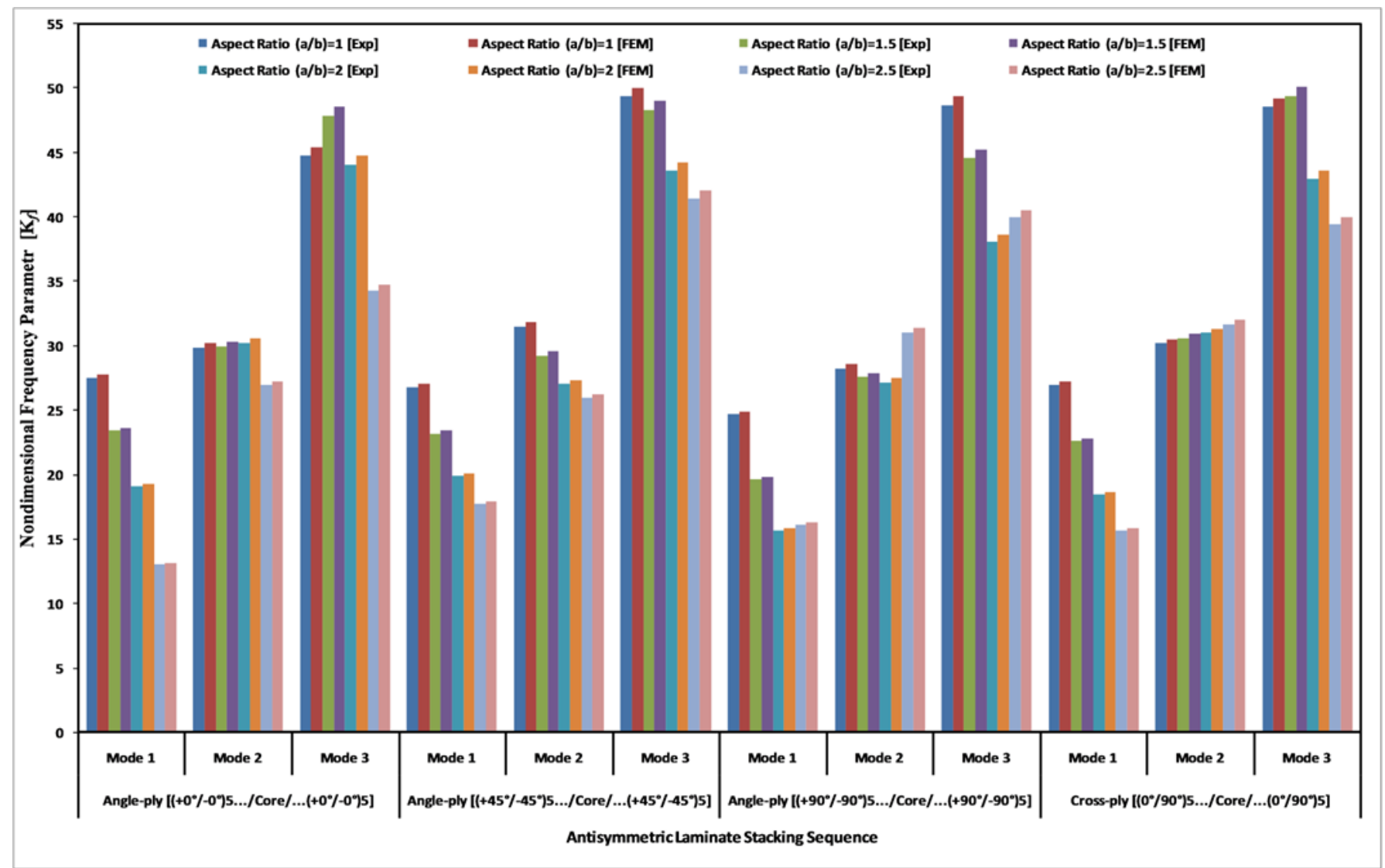

Fig. 4. Variation of $\mathrm{Kf}$ with $\mathrm{AR}(\mathrm{a} / \mathrm{b})$ for C-F-C-F laminated composite sandwich skew plates with skew angle $a=30^{\circ}$.

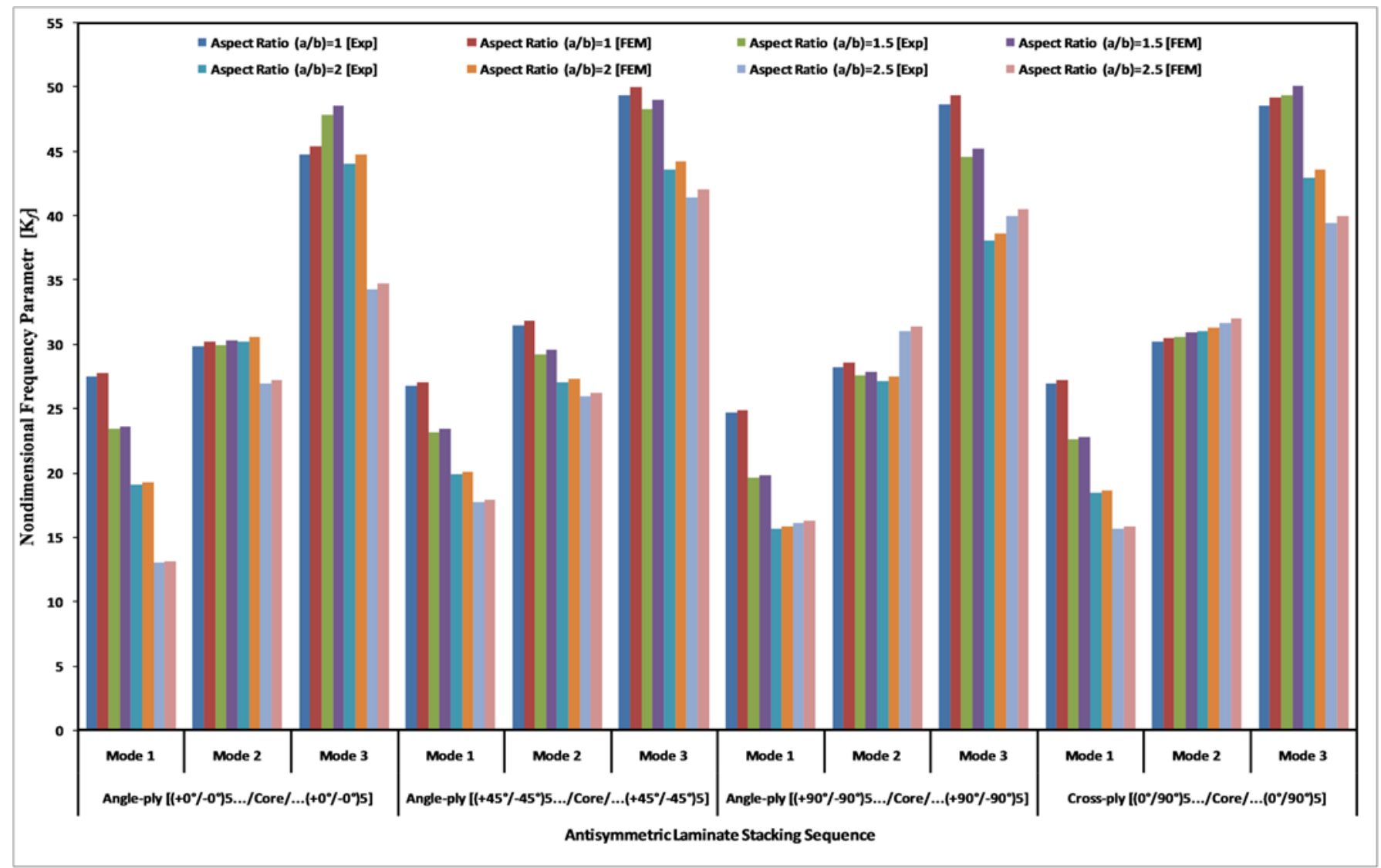

Fig. 5. Variation of $K_{f}$ with $A R(a / b)$ for C-F-C-F laminated composite sandwich skew plates with skew angle $a=45^{\circ}$. 


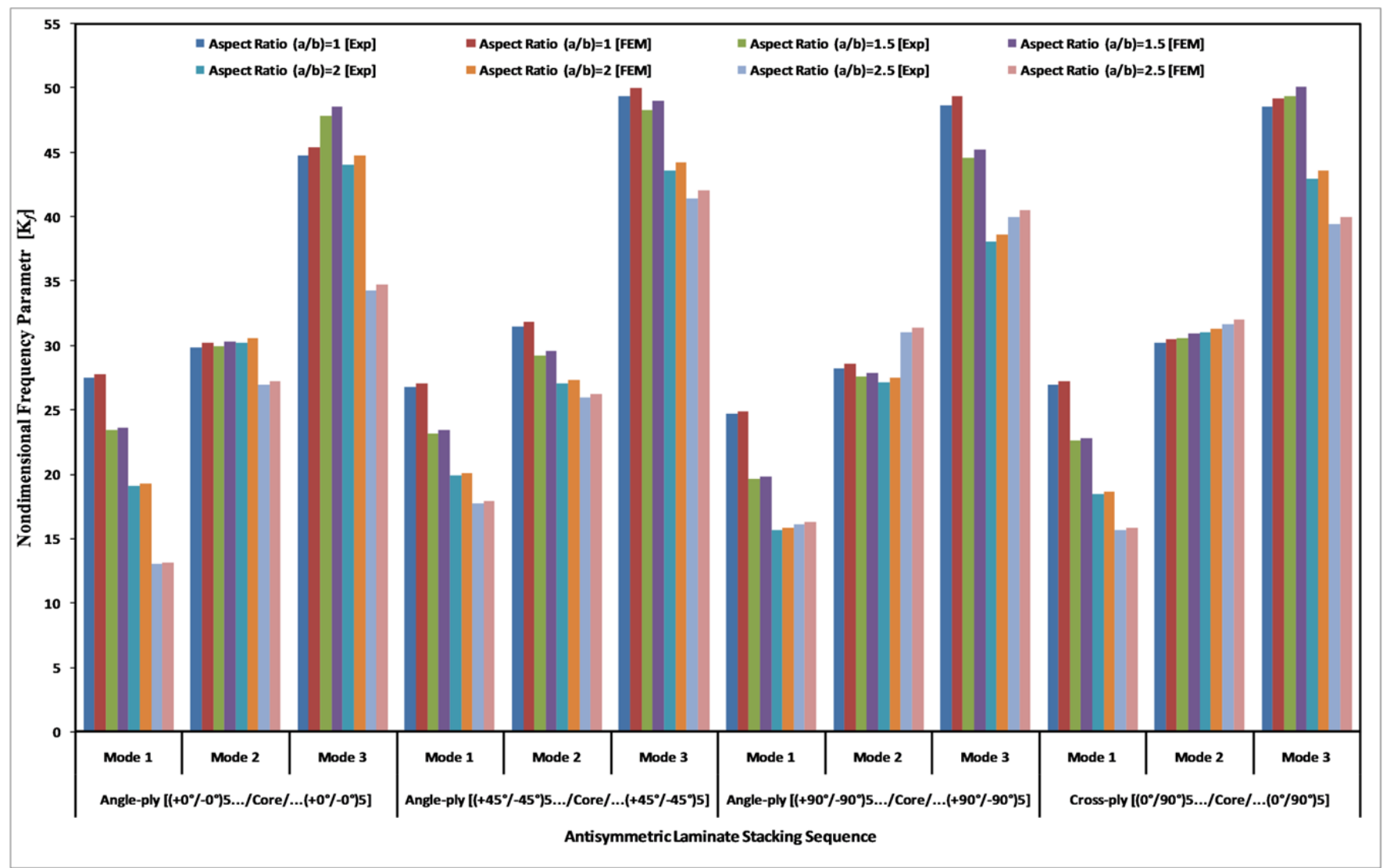

Fig. 6. Variation of $\mathrm{K}_{\mathrm{f}}$ with $\mathrm{AR}(\mathrm{a} / \mathrm{b})$ for C-F-F-F laminated composite sandwich skew plates with skew angle $a=0^{\circ}$.

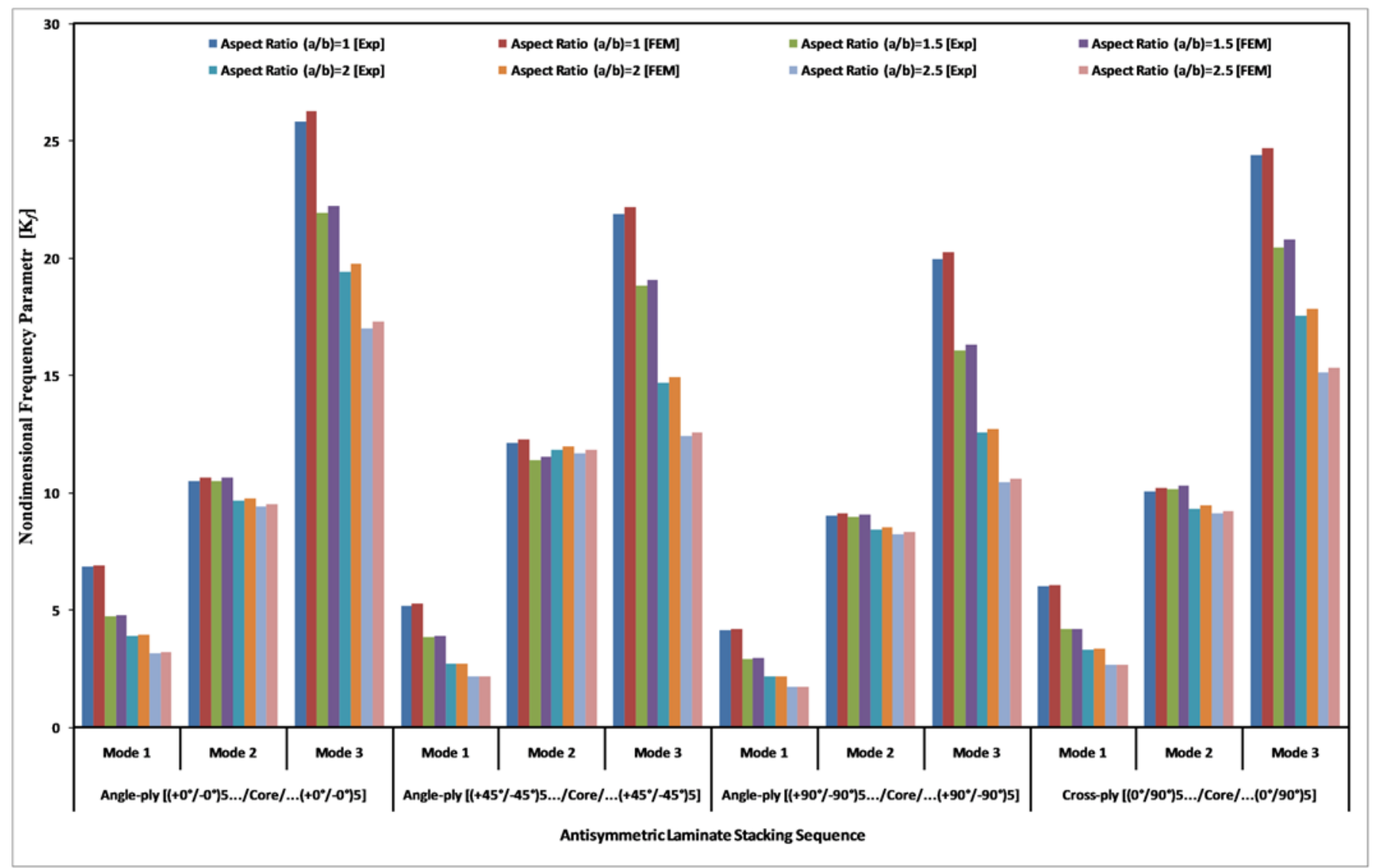

Fig. 7. Variation of $K_{f}$ with $A R(a / b)$ for C-F-F-F laminated composite sandwich skew plates with skew angle $a=15^{\circ}$. 


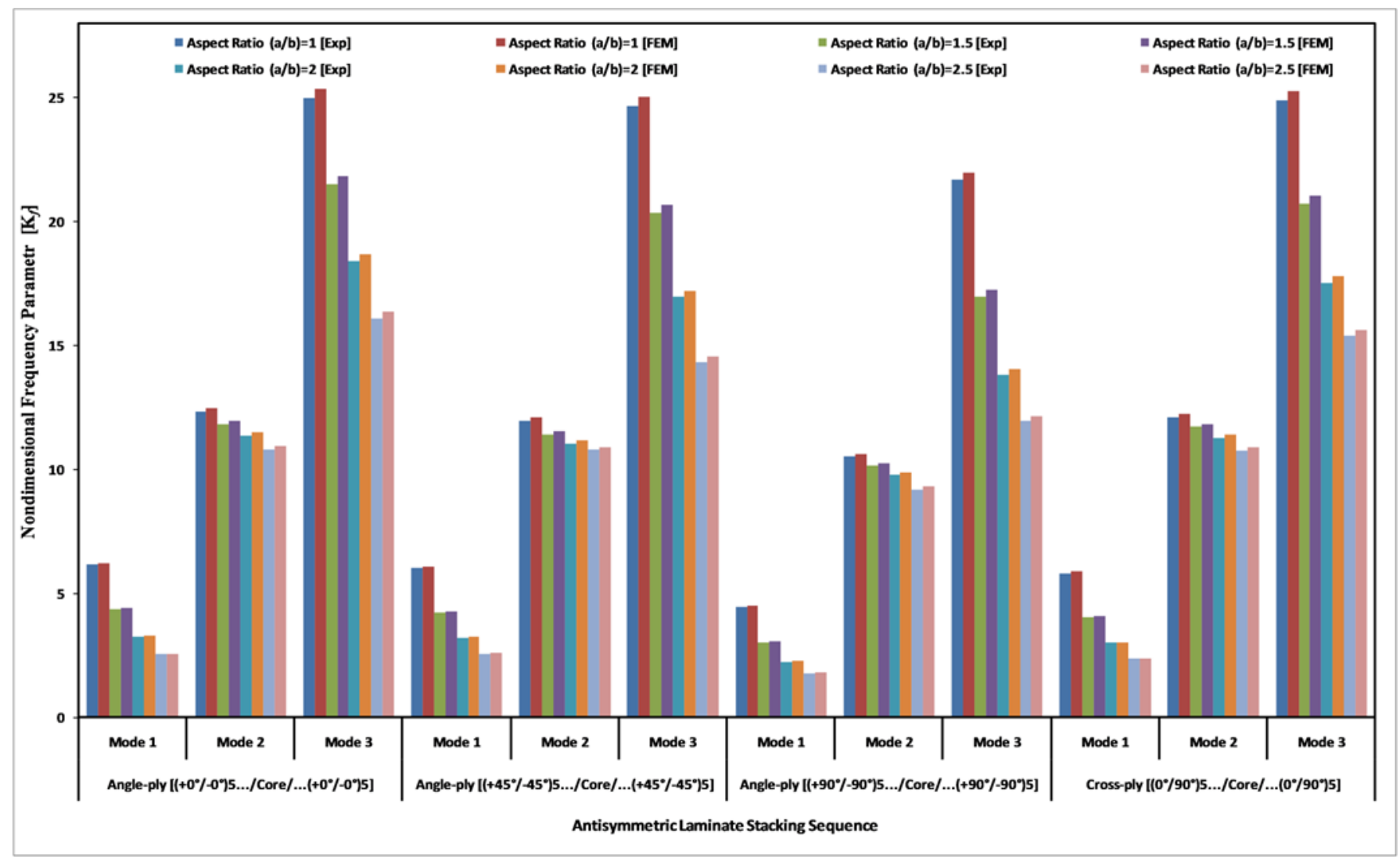

Fig. 8. Variation of $K_{f}$ with $A R(a / b)$ for C-F-F-F laminated composite sandwich skew plates with skew angle $a=30^{\circ}$.

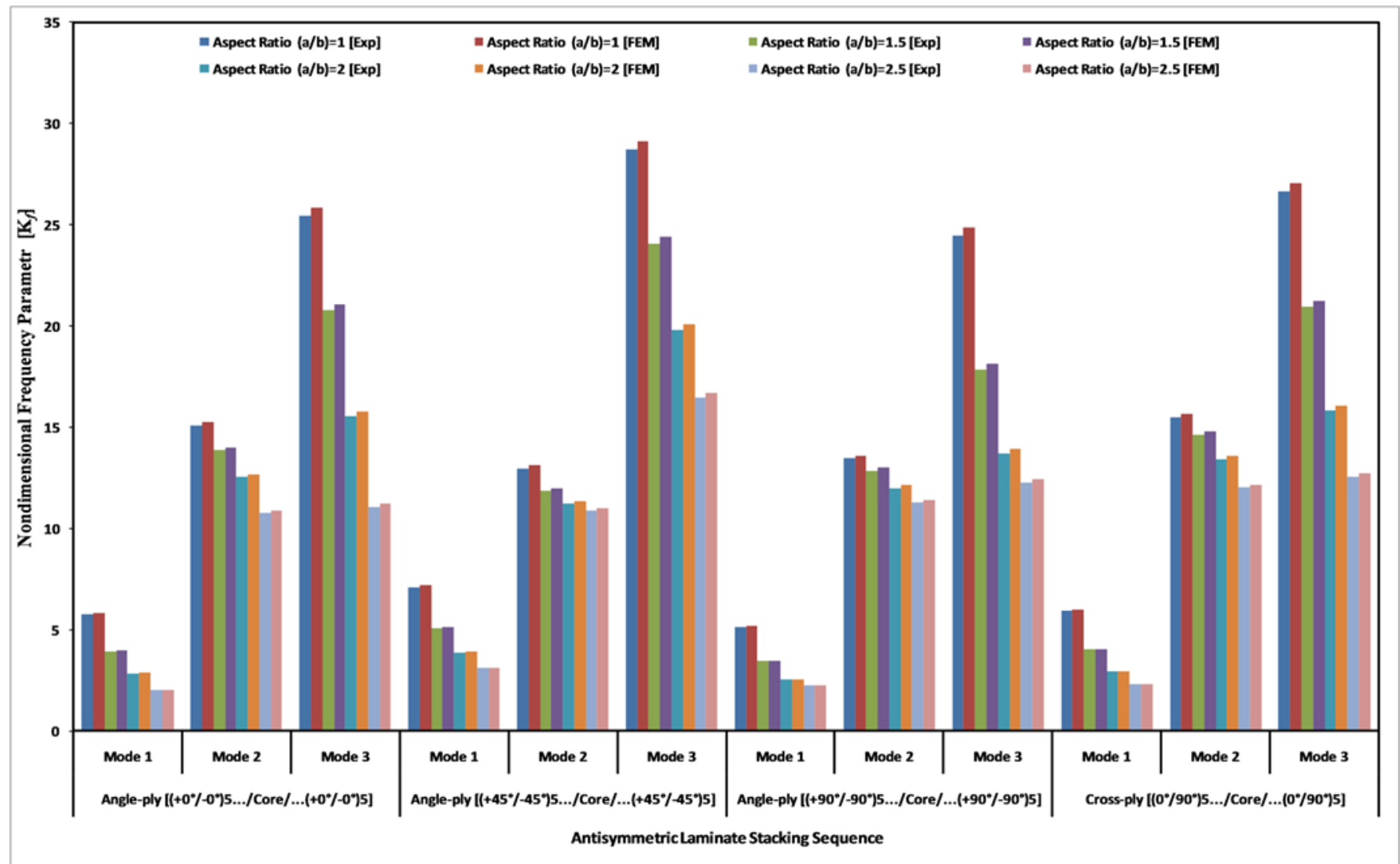

Fig. 9. Variation of $K_{f}$ with $A R(a / b)$ for C-F-F-F laminated composite sandwich skew plates with skew angle $a=45^{\circ}$. 


\section{sciendo}

Dhotre Pavan Kumar, Chikkol V. Srinivasa

Modal Characterization of Sandwich Skew Plates

Tab. 4. Mode shapes for C-F-C-F laminated composite sandwich skew plates $(a / b=1.5, N L=21$, antisymmetriccross-ply laminate)
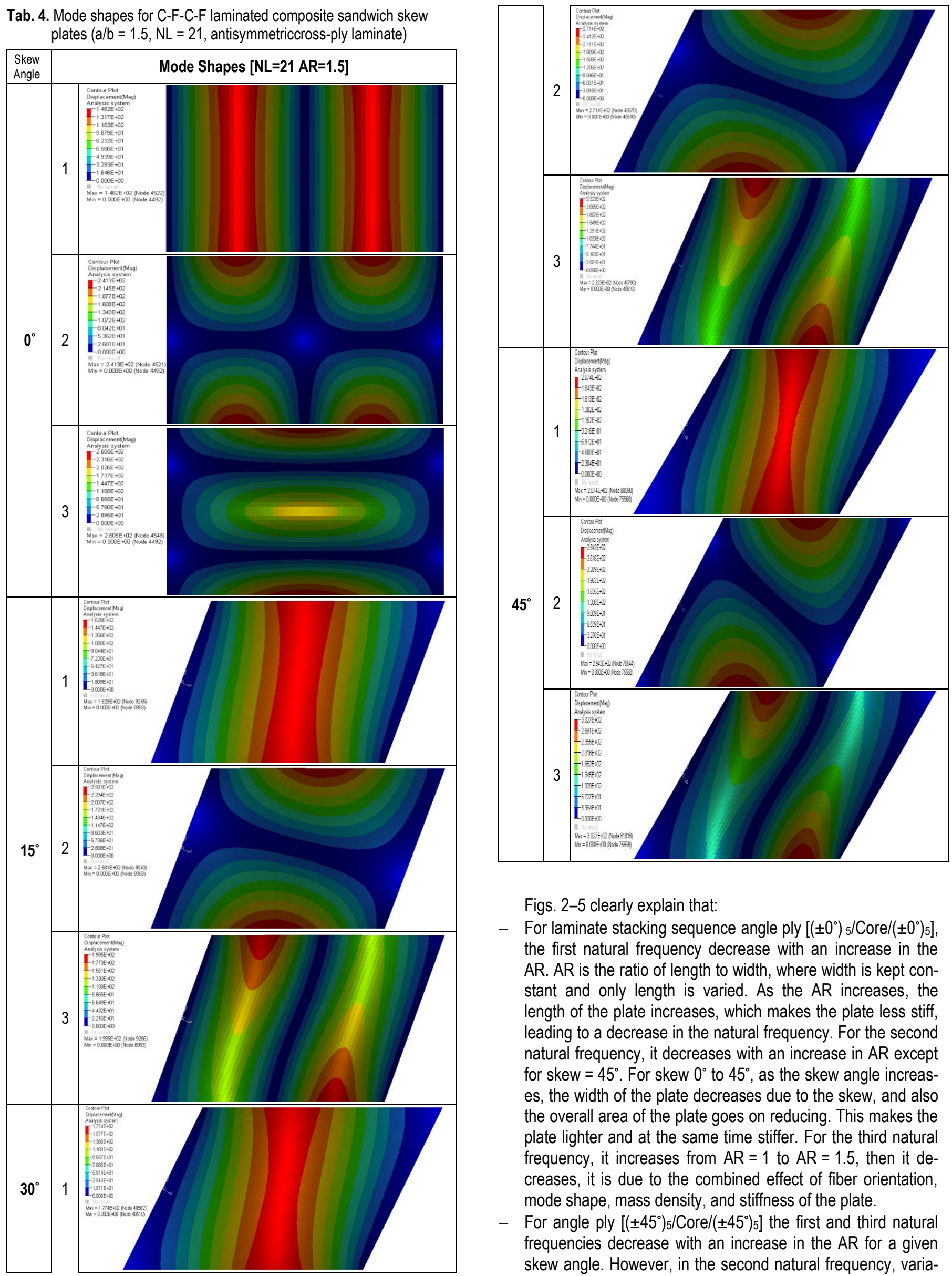

Figs. 2-5 clearly explain that:

- For laminate stacking sequence angle ply $\left[\left( \pm 0^{\circ}\right)_{5} / \mathrm{Core} /\left( \pm 0^{\circ}\right)_{5}\right]$, the first natural frequency decrease with an increase in the $A R . A R$ is the ratio of length to width, where width is kept constant and only length is varied. As the AR increases, the length of the plate increases, which makes the plate less stiff, leading to a decrease in the natural frequency. For the second natural frequency, it decreases with an increase in AR except for skew $=45^{\circ}$. For skew $0^{\circ}$ to $45^{\circ}$, as the skew angle increases, the width of the plate decreases due to the skew, and also the overall area of the plate goes on reducing. This makes the plate lighter and at the same time stiffer. For the third natural frequency, it increases from $A R=1$ to $A R=1.5$, then it decreases, it is due to the combined effect of fiber orientation, mode shape, mass density, and stiffness of the plate.

- For angle ply $\left[\left( \pm 45^{\circ}\right)_{5} /\right.$ Core $\left./\left( \pm 45^{\circ}\right)_{5}\right]$ the first and third natural frequencies decrease with an increase in the AR for a given skew angle. However, in the second natural frequency, variation is negligible for skew $=0^{\circ}$; it is considerable for other 
skew angles. Here the plate produces more stiffness due to the fiber orientation and it overshadows the effect of plate stiffness due to the change in the AR compared to other skew angles.

- In angle ply $\left[\left( \pm 90^{\circ}\right)_{5} /\right.$ Core $\left./\left( \pm 90^{\circ}\right)_{5}\right]$ stacking sequence, for all three frequencies, the $\mathrm{K}_{\mathrm{f}}$ value decreases as the increase in the $A R$ except skew $=45^{\circ}$. For skew $=45^{\circ}$ the $K_{f}$ value decreases from $A R=1$ to $A R=1.5$ and then it increases. It is exactly opposite as in the case of angle ply $\left[\left( \pm 0^{\circ}\right)_{5} /\right.$ Core $\left./\left( \pm 0^{\circ}\right)_{5}\right]$.

- The $K_{f}$ value decreases with an increase in the AR in all the three modes of cross-ply $\left[\left(0^{\circ} / 90^{\circ}\right)_{5} /\right.$ Core $\left./\left(0^{\circ} / 90^{\circ}\right)_{5}\right]$ stacking sequence from skew $=0^{\circ}$ to $30^{\circ}$. The only exception is for skew $=45^{\circ}$ in which the second natural frequency goes on increasing as the AR increased. The third natural frequency first increases from $A R=1$ to $A R=1.5$ and then decreases.

Tab. 5. Mode shapes for C-F-F-F laminated composite sandwich skew plates $(a / b=1.5, N L=21$, antisymmetric cross-ply laminate)

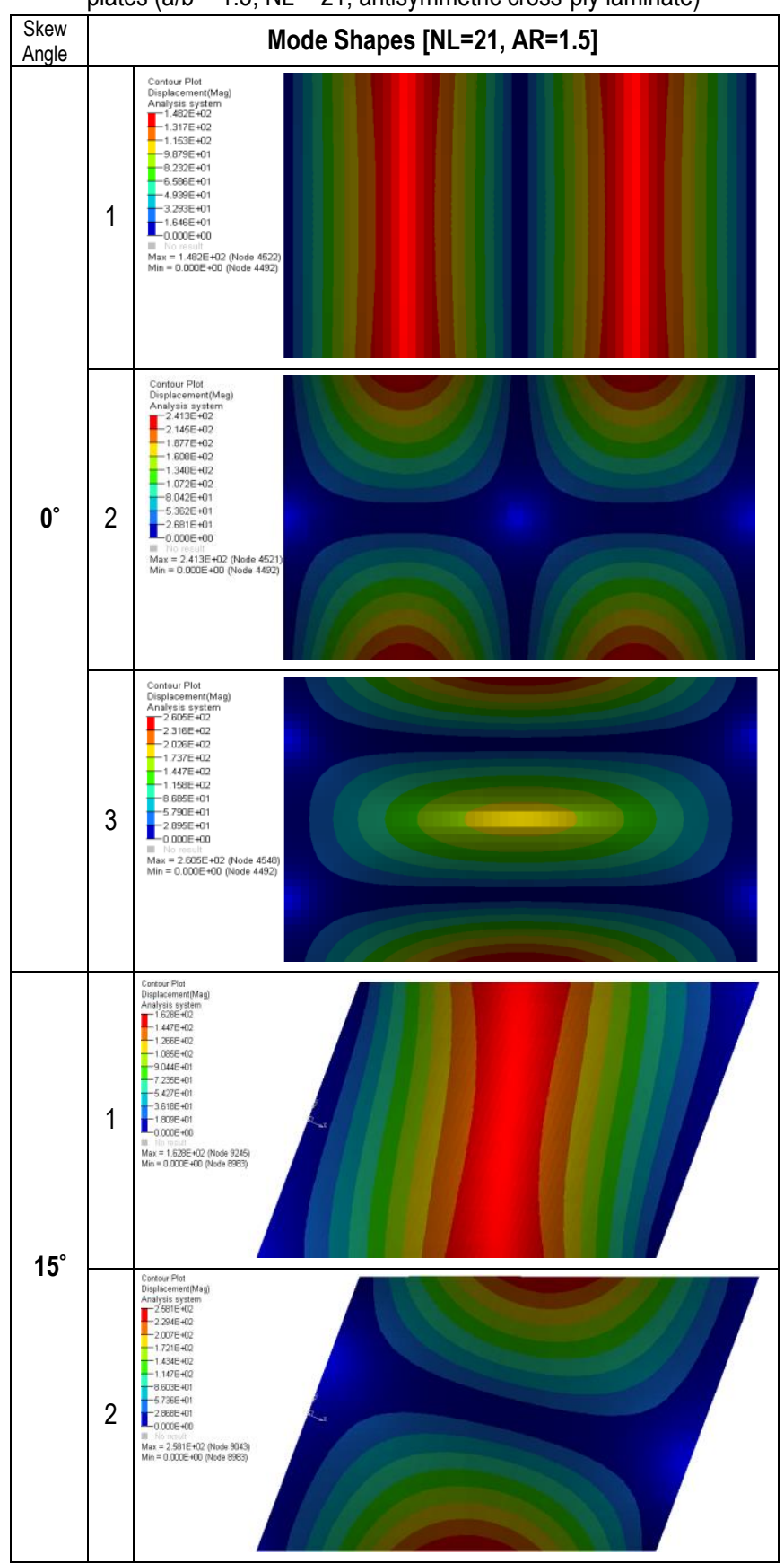

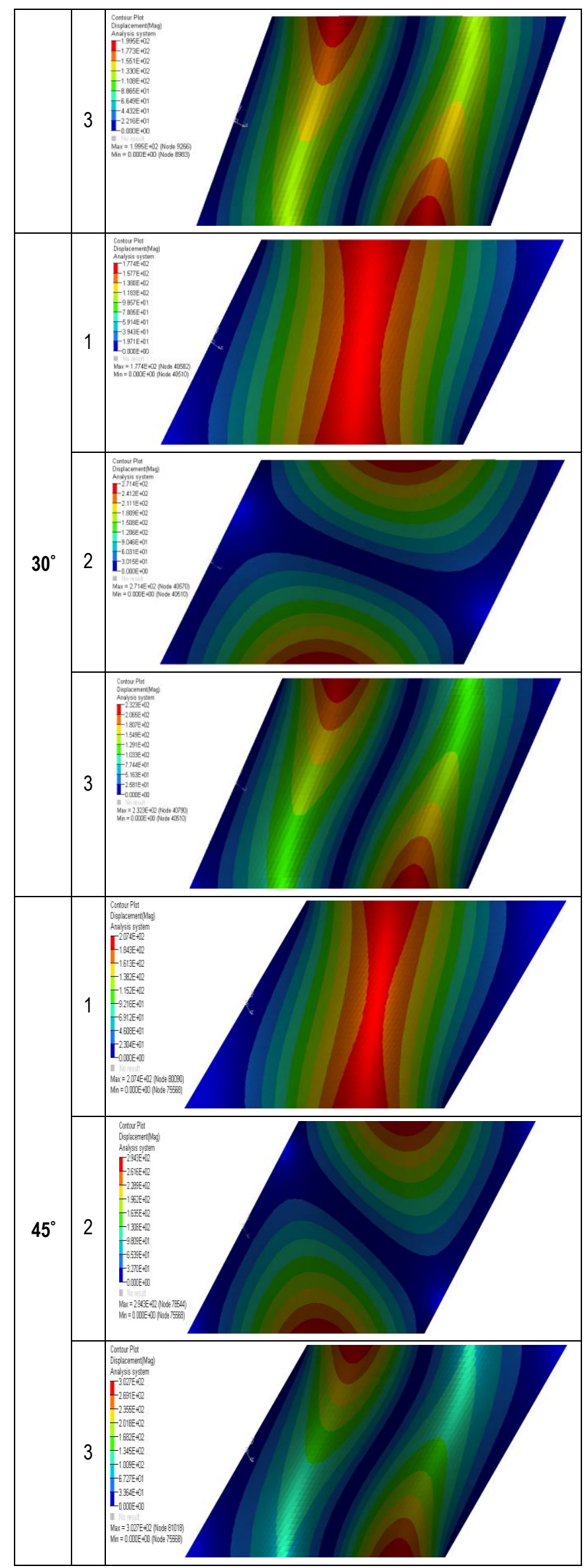


And from Figs. 6-9, the following is observed:

- With skew $=15^{\circ}$ for cross-ply $\left[\left(0^{\circ} / 90^{\circ}\right)_{5} /\right.$ Core $\left./\left(0^{\circ} / 90^{\circ}\right)_{5}\right]$ and Angle ply $\left[\left( \pm 45^{\circ}\right)_{5} / \mathrm{Core} /\left( \pm 45^{\circ}\right)_{5}\right]$, the second natural frequency decreases then it increases and it increases then it decreases is the only change.

- The $K_{f}$ value decreases in the order of laminate stacking sequence:angle ply $\left[\left( \pm 0^{\circ}\right)_{5} / \mathrm{Core} /\left( \pm 0^{\circ}\right)_{5}\right]$, cross-ply $\left[\left(0^{\circ} / 90^{\circ}\right)\right.$ $\left.{ }_{5} / \mathrm{Core} /\left(0^{\circ} / 90^{\circ}\right)_{5}\right]$, angle ply $\left[\left( \pm 45^{\circ}\right)_{5} / \mathrm{Core} /\left( \pm 45^{\circ}\right)_{5}\right]$ angle ply $\left[\left( \pm 90^{\circ}\right)_{5} /\right.$ Core $\left./\left( \pm 90^{\circ}\right)_{5}\right]$.

- For a given value of $A R$, as the skew angle is increased, the

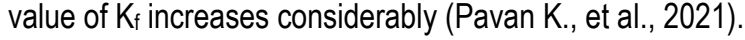

- The values of $\mathrm{K}_{f}$ are higher for C-F-C-F than C-F-F-F edge conditions for any given AR and skew angle.

- The experimental values in the form of the non-dimensional frequency coefficient $\mathrm{K}_{\mathrm{f}}$ are very close to accurate and promising to those of the finite element solution.

\section{CONCLUSION}

Free vibration investigation was made on laminated sandwich skew plates adopting both experimental and finite element methods. Glass epoxy laminated composites are used as face sheets and aluminum honeycomb (Al3003) is used as the core in the current study. Two types of edge conditions were used. i.e., C-F$\mathrm{C}-\mathrm{F}$ and $\mathrm{C}-\mathrm{F}-\mathrm{F}-\mathrm{F}$. The experimentally obtained results were then validated by finite element values, and the experimental values are promising and close to the finite element values. Influences of various parameters, for instance, skew angle, AR, laminate stacking sequence, and edge conditions are studied. The thickness ratio of core to face sheet $t_{c} / t_{f}=6$ was kept constant all through the study. As the AR increases for any skew angle, the $K_{f}$ value decreases irrespective of the edge condition. When the AR increases, the length of the sandwich plate also increases. This reduces the stiffness of the sandwich skew plate. The skew angle plays a major role in the dynamic response of the sandwich skew plate. When the skew angle is increased, the $K_{f}$ value increases for all ARs. The fiber orientation of the lamina plays a significant role in deciding the vibration response of the sandwich skew plates. When the fiber angle is $0^{\circ}$ i.e., the fiber is placed parallel to the length of the plate, the plate produces higher natural frequency due to more stiffness in the longitudinal direction. In addition, the lowest natural frequency is observed when the fiber angle is $90^{\circ}$ i.e., the fiber angle is perpendicular to the length of the plate. The natural frequencies for the other laminate stacking sequences lie in between these two extreme values. The sandwich skew plates with C-F-C-F edge conditions produce a higher natural frequency than plates with C-F-F-F edge conditions.

\section{REFERENCES}

1. Abdi B., Azwan S., Ayob A., Rahman R.A., Abdullah R.A. (2014), Experimental Investigation on Free Vibration of Foam-Core Sandwich Plate with and without Circular Polymer Columns, Advanced Materials Research , 845, 297-301.

2. Adarsh K., Ramesh S.S. (2015), Modal Analysis and Testing of Honeycomb Sandwich Composites, Topics in Modal Analysis, Volume 10, Proceedings of the 33rd IMAC, A Conference and Exposition on Structural Dynamics, Springer International Publishing, 237-241.
3. Aman G., Chalak H.D. (2019), A review on analysis of laminated composite and sandwich structures under hygrothermal conditions, Thin-Walled Structures, 142, https://doi.org/10.1016/j.tws.2019.05. 005.

4. Aman G., Chalak H.D. (2020), Analysis of non-skew and skew laminated composite and sandwich plates under hygro-thermo mechanical conditions including transverse stress variations, Journal of Sandwich Structures and Materials, DOI: 10.1177/109963622093 2782.

5. Aman G., Mohamed O.B., Chalak H.D., Anupam C. (2020), A review of the analysis of sandwich FGM structures, Composite Structures, https://doi.org/10.1016/j.compstruct.2020.113427.

6. Andena L., Manconi E., Manzoni S., Moschini S., Vanali M. (2012), Experimental tests and numerical modeling of a sandwich panel, 25th International Conference on Noise and Vibration engineering (ISMA2012), 4th International Conference on Uncertainty in Structural Dynamics (USD2012), Leuven.

7. Arunkumar M.P., Jeyaraj P., Ganga Dharan K.V., Surya Narayana Reddy C.V. (2020), Numerical and experimental study on dynamic characteristics of honeycomb core sandwich panel from equivalent 2D model, Sadhana, 45, 206, 1-6.

8. Barkanov E., Chate A., Skukis E., Täger O., Kolsters H. (2005), Finite element and experimental vibration analysis of viscoelastic composite structures, Computational Methods and Experimental Measurements XII, 41, 527-537.

9. Benjeddou A., Guerich M. (2019), Free vibration of actual aircraft and spacecraft hexagonal honeycomb sandwich panels: A practical detailed FE approach, Advances in Aircraft and Spacecraft Science, 6(2), 169-187.

10. Berthelot J.M., Assarar M., Sefrani Y., Mahi A.E. (2008), Damping analysis of composite materials and structures, Composite Structures, 85, 189-204.

11. Chang J.S., Chen H.C., Lin H.T. (2006), Numerical and experimental studies on aluminum sandwich plates of variable thickness, Journal of the Chinese Institute of Engineers, 29(5), 851-862.

12. Jones R.M. (1999), Mechanics of Composite Materials, Taylor and Francis.

13. Jun L.,Congkuan H., Wenbin Y., Fan Y., Gao L. (2021), Free vibration and transient dynamic response of functionally graded sandwich plates with power-law nonhomogeneity by the scaled boundary finite element method, Computer Methods in Applied Mechanics and Engineering, 376, https://doi.org/10.1016/j.cma. 2021.113665

14. Lee C.R., Kam T.Y., Sun S.J. (2007), Free-Vibration Analysis and Material Constants Identification of Laminated Composite Sandwich Plates, Journal of Engineering Mechanics, 133 (8), 874-886.

15. Maheri M.R., Adams R.D., Hugon J. (2008), Vibration damping in sandwich panels, Journal of Material Science, 43, 6604-6618.

16. Mondal S., Patra A.K., Chakraborty S., Mitra N. (2015), Dynamic performance of sandwich composite plates with circular hole/cut-out: A mixed experimental-numerical study, Composite Structures, 131, 479-489.

17. Pavan K., Srinivasa C.V. (2020), Free vibration studies on skew sandwich plates by FEM, IOP Conference Series: Materials Science and Engineering, 925, 012024, Doi:10.1088/1757-899X/925/1/ 012024.

18. Pavan K., Srinivasa C.V. (2020), On buckling and free vibration studies of sandwich plates and cylindrical shells: A review, Journal of thermoplastic composite materials, 33 (5), 1-51.

19. Pavan K.D., Srinivasa C.V. (2021), On free vibration of laminated skew sandwich plates: A Finite element analysis, Nonlinear Engineering, https://doi.org/10.1515/nleng-2021-0006, 2021

20. Petrone G., Alessandro V.D., Franco F., Mace B., De Rosa S. (2014), Modal characterisation of recyclable foam sandwich panels, Composite Structures, 113, 362-368.

21. Prasad E.V., Sahu S.K. (2018), Vibration Analysis of Woven Fiber Metal Laminated Plates - Experimental and Numerical Studies, 
International Journal of Structural Stability and Dynamics, 18, 1850144-1-23.

22. Rajkumar S., Ravindran D., Ramesh S.S., Raghupathy V.P. (2014), Evaluation of elastic constants of A3003 honeycomb core with varying hexagonal cell geometries through finite element approach, Proceedings of the Institution of Mechanical Engineers Part C Journal of Mechanical Engineering Science, 203-210, DOI: 10.1177/0954406213510491.

23. Raville M.E., Ueng C.E.S. (1967), Determination of Natural Frequencies of Vibration of a Sandwich Plate, Experimental Mechanics, 7, 490-493, https://doi.org/10.1007/BF02326265.

24. Rezvani S.S., Kiasat M.S. (2018), Analytical and experimental investigation on the free vibration of a floating composite sandwich plate having viscoelastic core, Archives of Civil and Mechanical Engineering, 18, 1241-1258.

25. Su Bin L., Chang-Yong. L., Dewey H.H. (2020), On the mechanics of composite sandwich plates with three-dimensional stress recovery, International Journal of Engineering Science, 157.

26. Vinayak K., Balaji K., Kattimani S.C. (2020), Effect of temperature and moisture on free vibration characteristics of skew laminated hybrid composite and sandwich plates, Thin-Walled Structures, 157, https://doi.org/10.1016/j.tws.2020.107113.
27. Yang J.S., Xiong J., Ma L., Wu L.Z. (2014), Vibration and damping performances of carbon fiber composite pyramidal truss sandwich panels embedded with viscoelastic layers, ECCM16 - 16TH European conference on composite materials, Seville, Spain, 22-26.

28. Zhicheng H., Xingguo W., Nanxing W., Fulei C., Jing L. (2020), The Finite Element Modeling and Experimental Study of Sandwich Plates with Frequency-Dependent Viscoelastic Material Model, Materials, 13, 2296, Doi:10.3390/ma13102296.

29. Zhuang L. (2006), Vibration and acoustical properties of sandwich composite, Degree of Doctor of Philosophy, Auburn University, Auburn, Alabama.

Acknowledgments: The authors would like to express gratitude to the Principal and Management of GM Institute of Technology, India, for their inspiration and support provided.

Dhotre Pavan Kumar: (D https://orcid.org/0000-0002-7900-755X

Chikkol V. Srinivasa: (D) https://orcid.org/0000-0002-9794-3886 